\title{
Linx
}

Revue des linguistes de l'université Paris X Nanterre

$61 \mid 2009$

Entre rection et incidence : des constructions verbales atypiques?

\section{Grammaire et dictionnaire : le parti pris des mots}

\section{Annie Bertin}

\section{(2) OpenEdition}

\section{Journals}

Édition électronique

URL : http://journals.openedition.org/linx/1332

DOI : $10.4000 / \operatorname{linx} .1332$

ISSN : 2118-9692

Éditeur

Presses universitaires de Paris Nanterre

\section{Édition imprimée}

Date de publication : 1 juin 2009

Pagination : 71-85

ISSN : 0246-8743

\section{Référence électronique}

Annie Bertin, «Grammaire et dictionnaire : le parti pris des mots », Linx [En ligne], 61 | 2009, mis en ligne le 01 juin 2013, consulté le 21 avril 2019. URL : http://journals.openedition.org/linx/1332 ; DOI : $10.4000 / \operatorname{linx} .1332$ 


\title{
Les parenthétiques comme «Unités Illocutoires Associées ». Une perspective macrosyntaxique
}

\author{
Sylvain Kahane \\ Modyco, Université Paris Ouest Nanterre \& CNRS \\ Paola Pietrandrea \\ Lattice, ENS \& CNRS / Università Roma Tre
}

\section{Introduction}

Dans cet article, nous allons nous intéresser aux parenthétiques, c'est-à-dire aux clauses réduites, comme je crois en (1), qui comportent un verbe transitif dont le deuxième argument sémantique, habituellement réalisé par une complétive objet, est ici une phrase à part entière dans laquelle la parenthétique est insérée ( $c f$. par exemple la définition de Schneider (2007, pp. 77-78) que nous commenterons plus loin) :

(1) a. nous avons attendu je crois sept ou huit heures dans la gare parce que tous les officiers allemands tout l'état major allemand rentrait à Paris // (D003) ${ }^{1}$

b. mes souvenirs les plus anciens sont je crois des souvenirs de Mulhouse sûrement pas de Cannes // (D204)

\footnotetext{
${ }^{1}$ Nos conventions d'annotation des exemples sont présentées dans la section 3.1. Le numéro indique l'échantillon du corpus Rhapsodie dont l'exemple est extrait.
} 
Nous étendrons cette notion aux clauses réduites lorsqu'elles sont en position initiale ou finale et ne sont pas à proprement parler « parenthétiques » :

(2) a. du square des postes je peux rejoindre la place de l'Etoile // et là j'ai deux itinéraires je crois // ou bien je prends la rue Lesguidières et le boulevard Edouard Rey // et non c'est plus Edouard Rey c'est Emmanu c'est fils de la Sarce je crois // non c'est même pas ça // je m'embrouille // (M011)

b. ça te va bien // ça te va bien tu sais // (D207)

c. Ha non je me souviens maintenant, c'est mon gendre qui a habité en Martinique quelques années qui m'avait fait voir un film... et m'avait expliqué cet étrange arbre... (commentaire sur un blog)

Nous abordons le problème de la caractérisation et de la description de ces objets dans le cadre de l'approche macrosyntaxique héritée de l'école d'Aix et tout particulièrement des travaux de Blanche-Benveniste et al. (1990) et de Deulofeu (1999). La particularité principale de cette approche est de considérer deux niveaux de cohésion syntaxique : un premier niveau, appelé microsyntaxe, considère uniquement les segments où les éléments sont reliés par des relations de rection, tandis que la macrosyntaxe couvre tous les phénomènes montrant de la cohésion syntaxique sans qu'il y ait pour autant rection.

Comme l'a noté Blakemore (2006), la description des parenthétiques suppose que l'on précise à quel niveau de structuration elles peuvent être considérées comme indépendantes et à quel niveau de structuration elles peuvent être considérées comme intégrées à la phrase sur laquelle elles s'ancrent. La littérature propose essentiellement deux solutions différentes, reposant sur une conception globale de l'organisation linguistique. Une partie de la littérature retient que le lien entre la parenthétique et son ancre est un lien de nature strictement syntaxique que l'on peut caractériser par une série de transformations et d'effacements (Ross 1973, Schelfhout et al. 2004, Dehé \& Kavalova 2006). D'autres approches retiennent au contraire que la parenthétique et son ancre sont à considérer comme des clauses syntaxiquement indépendantes l'une de l'autre et que leur intégration sémantique découle d'un processus inférentiel qui se produit au niveau de l'interprétation de l'énoncé (Ifantidou 2001, Espinal 1991, Haegeman 1988). Notre approche macrosyntaxique permet de concilier ces différentes approches en donnant à la relation entre la parenthétique et son ancre une réalité syntaxique sans pour autant devoir la considérer comme une relation de rection microsyntaxique ( $c$. aussi Iordanskaja \& Mel'čuk 2011). Nous montrerons également que la relation macrosyntaxique qu'entretiennent les parenthétiques avec leur ancre concerne plus largement une classe d'unités syntaxiques que nous appellerons les unités illocutoires associées (UIA), dont nous donnerons les principales propriétés syntaxiques et sémantiques et dont nous proposerons une classification fonctionnelle.

Dans cet article, nous commencerons par montrer dans les sections 2 et 3 en quoi les parenthétiques relèvent bien de la macrosyntaxe et ce qui les distingue des autres relations macrosyntaxiques décrites dans la littérature. Nous expliquerons pourquoi nous proposons d'appeler les segments qui nous intéressent des Unités Illocutoires Associées et nous verrons que l'on peut également considérer parmi les UIA des segments non verbaux de type marqueurs de discours. Dans les sections 4 et 5 , nous 
étudierons les propriétés sémantiques de la relation entre l'UIA et son contexte. Ceci nous amènera dans la section 6 à la définition des UIA, à la comparaison avec la définition des parenthétiques et à la justification de considérer une autre classe plus étendue incluant notamment des marqueurs de discours. Nous verrons dans la section 7 que la classe des UIA se justifie également sur des bases fonctionnelles.

La plupart de nos exemples sont tirés d'un corpus de 3 heures (33 000 mots) en cours d'annotation syntaxique et prosodique dans le cadre du projet ANR Rhapsodie ${ }^{2}$.

\section{Parenthétiques et microsyntaxe}

\subsection{Microsyntaxe et unité rectionnelle}

Nous considérons qu'un segment entretient une relation syntaxique avec son contexte quand s'exercent sur ce segment des contraintes distributionnelles sur le paradigme des éléments qui peuvent commuter avec lui, sur les insertions et suppressions qui peuvent être effectuées dans ce segment et les segments adjacents et sur les déplacement possibles de ce segment.

On peut considérer plusieurs niveaux de cohésion syntaxique.

Le niveau de cohésion syntaxique le plus étroit que nous considérons est la rection. Un segment est dit régi lorsque sa distribution est contrôlée par un autre élément et qu'il se voit imposer des parties du discours particulières avec des marqueurs morphologiques ou analytiques précis et notamment la commutation avec des pronoms porteurs de marques casuelles ( $c f$. les tests habituels pour identifier un sujet par la commutation avec il ou qui est-ce qui).

(3) l'équipe de France n'arrive pas à récupérer ce ballon. $\rightarrow$ elle n'y arrive pas.

Parmi les critères indiquant la rection par un verbe, nous retenons la possibilité d'être clivé. Ainsi le segment à vive allure dans l'exemple (4)a est-il considéré comme régi, tandis que le segment à mon avis dans l'exemple (5)a est considéré comme non régi :

(4) a. il traversa la place à vive allure.

b. c'est à vive allure qu'il traversa la place.

(5) a. il n'a pas traversé la place à mon avis.

b. *c'est à mon avis qu'il n'a pas traversé la place.

Dans la suite, nous appellerons unités rectionelles (UR) les segments maximaux dont tous les mots sont reliés par des relations de rection (Benzitoun et al. 2010). Dans l'exemple (5)a, il y a donc deux UR : à mon avis et il n'a pas traversé la place.

Nous étendons la notion d'UR aux entassements, c'est-à-dire aux segments qui occupent la même place rectionnelle qu'un segment précédent. Il peut s'agir d'une

\footnotetext{
2 Projet financé par l'Agence Nationale de la Recherche (contrat ANR Rhapsodie 07 Corp-030-01, Corpus prosodique de référence du français parlé, dirigé par Anne Lacheret).
} 
coordination ( $c f .(6) a)$, d'une reformulation ( $c f .(6) b)$, ou de certains phénomènes d'exemplification pour lesquels une discontinuité de l'entassement est possible (cf. (6)c) (Gerdes \& Kahane 2009, Kahane \& Pietrandrea à par.).

a. des jeunes $\left\{\right.$ cagoulés $\left.\right|^{\wedge}$ et armés $\}$ ont $\{$ pillé des magasins | brûlé $\{$ des pneus |^et des voitures \} \} // (M206)

b. je voulais pas aller à Addis Abeba // puisque $\{\{\{$ les | les | les | les $\}$ c $\sim$ les capitales \} | les grandes villes $\}$ ne me disaient rien du tout // (D204)

c. si je ne craignais pas d'entrer dans le jeu de certains hommes qui abusent de leur condition je dirais que vous avez donné $\{$ quelque chose de plus $\mid\}$ à la femme $\{\mid$ des armes de persuasion $\} / /(\mathrm{D} 201)$

Les différents segments qui viennent occuper une même place syntaxique forment les couches d'un entassement et héritent de la relation de rection de la première couche (Kahane à par.). Nous verrons par la suite ( $c f .7)$ que les parenthétiques peuvent jouer un rôle saillant dans la construction des entassements.

L'UR est par définition le domaine de la microsyntaxe et tous les phénomènes syntaxiques au delà de l'UR appartiennent à la macrosyntaxe.

\subsection{Parenthétiques et rection}

Nous allons maintenant étudier les parenthétiques. Reprenons notre exemple (1b) sous (7) :

(7) j’ai deux itinéraires je crois

On peut être tenté d'y voir une relation de rection, notamment parce que cet exemple (comme il a été remarqué par plusieurs auteurs, à partir d’Urmson 1952) peut être paraphrasé par :

(8) a. je crois que j’ai deux itinéraires

Mais lorsque j'ai deux itinéraires est en position d'objet canonique, la conjonction est requise en français (bien que je crois j'ai deux itinéraires ne semble pas être une production impossible), alors qu'elle est impossible dans le cas de la parenthétique :

b. *que j'ai deux itinéraires je crois

Ceci contraste avec les cas d'antéposition d'une complétive objet où la conjonction est obligatoire comme le montre le test emprunté à Blanche-Benveniste \& Willems (2007 : 244) :

(9) a. que tu fasses ça j'aime pas

b. *tu fasses ça j’aime pas

Par ailleurs, en l'absence de la conjonction, le semi-clivage est impossible (cf. (10)c) :

(10) a. ce que j'aime, c'est le chocolat

b. ce que je crois, c'est que j'ai deux itinéraires

c. *ce que je crois, c'est j'ai deux itinéraires 
Nous en concluons qu'il n'y a pas dans l'exemple (7) de relation de rection entre les deux segments je crois et j'ai deux itinéraires et qu'il s'agit donc de deux UR. Nous considérons néanmoins qu'il y a une relation syntaxique entre les deux UR que nous allons continuer à étudier.

Nous appellerons ancre de la parenthétique l'UR à laquelle la parenthétique est associée, à l'instar de Brinton (2007).

\section{Parenthétiques et macrosyntaxe}

\subsection{Unité illocutoire, noyau et adnoyau}

Considérons l'énoncé :

(11) a. moi < ma mère < le salon < c'est de la moquette // (CorpAix)

Bien que cet énoncé soit constitué de quatre UR, on reconnait une certaine cohésion, due au fait que ces quatre UR encodent un seul acte illocutoire, une seule assertion. Nous dirons qu'il s'agit d'une unité illocutoire (UI) (cf. Benzitoun et al. (2010) pour une caractérisation de cette notion).

Parmi ces quatre UR, un segment encode à lui seul la force illocutoire ( $c$. Cresti 2000 ) et peut recevoir des modalités d'énonciation telle que la négation ou l'interrogation :

b. moi < ma mère < le salon < c'est pas de la moquette //

c. toi $<$ ta mère < le salon < est-ce que c'est de la moquette ? //

Ce segment est appelé le noyau de l'UI. Les autres UR, dépourvues de force illocutoire, s'associent au noyau et sont appelées des adnoyaux ou plus précisément des prénoyaux, in-noyaux ou post-noyaux selon leur position par rapport au noyau.

Nous adoptons les conventions d'annotation de Rhapsodie (Benzitoun et al. 2010) : // indique la frontière de l'UI, ce qui est avant < est un prénoyau et ce qui est après $>$ est un postnoyau. Les parenthétiques et les marqueurs de discours seront mis en italique.

A l'instar de Benzitoun et al. (2010), nous considérons que microsyntaxe et macrosyntaxe constituent deux niveaux d'organisation syntaxique assez indépendants l'un de l'autre. Certains phénomènes de rection, c'est-à-dire microsyntaxique, peuvent par exemple relier des éléments qui sont par ailleurs distribués sur des unités macrosyntaxiques différentes. C'est le cas par exemple de (12), étudié par Sabio (2006), où une relation de rection microsyntaxique relie un objet (deux euros) et une construction verbale (ça cô̂te) qui constituent respectivement le noyau et le post-noyau d'une même UI (le symbole + indique qu'une balise qui marque par défaut une frontière d'UR n'en est pas une) :

(12) a. deux euros >+ ça coûte // (entendu sur un marché)

Les relations microsyntaxiques peuvent également se poursuivre à travers des changements de tours de parole, lorsqu'un locuteur s'appuie sur l'UI de son interlocuteur pour produire la sienne : 
(13) A : donc $<$ moi < ben je vais je je prends le mét $\sim$ je prends le métro le matin bon jusqu' au Palais Royal //+

$\mathrm{B}$ : à quelle heure excusez-moi //

A : oui oui je prends le métro le matin à huit heures et demie // (D001)

Nous considérons que ces deux premiers tours de paroles, qui sont clairement deux UI alternant une assertion et une interrogation, forment ensemble une seule UR où la question de B s'appuie sur l'assertion de A en se plaçant dans la rection du verbe. Nous verrons que les "parenthétiques » peuvent fort bien former également des tours de paroles séparés de leur ancre.

\subsection{Parenthétiques et modalités d'énonciation: les Unités Illocutoires Associées}

Nous avons montré que la parenthétique n'entretient pas de relation de rection avec son ancre. Il nous reste à montrer qu'il y a bien une relation (macro)syntaxique entre les deux et déterminer s'il s'agit ou non d'une relation noyau-adnoyau.

Considérons les deux faits suivants : (i) l'UR parenthétique peut accepter des modalités d'énonciation, comme le montrent les exemples en (14), mais (ii) des contraintes s'imposent sur les modalités d'énonciation acceptées par les UR parenthétiques ( $c f .(15))$.

(14) a. c'est fils de la Sarce je crois. (M011)

b. c'est fils de la Sarce, tu crois?

c. c'est fils de la Sarce, tu crois pas?

(15) a. * c'est fils de la Sarce, je crois pas. ${ }^{3}$

b. * c'est fils de la Sarce, tu crois.

Ces contraintes qui s'exercent sur les modalités d'énonciation des parenthétiques sont le signe d'une dépendance syntaxique et donc d'une relation macrosyntaxique avec son ancre. La possibilité pour la parenthétique de recevoir tout de même des modalités d'énonciation, et notamment d'être interrogative quand son ancre est déclarative, prouve qu'elle n'est pas un adnoyau, mais plutôt un noyau secondaire. Elle forme donc une sorte d'UI en partie dépendante de l'UI ancre. C'est pour ça que nous décidons d'appeler les «parenthétiques » (qui comme nous l'avons déjà souligné ne forment pas nécessairement une parenthèse au sein de leur ancre) des Unités Illocutoires Associées (UIA).

\footnotetext{
${ }^{3}$ Une négation est possible lorsqu'elle fait écho à une négation dans l'ancre : non ça n'aide pas les élèves pour l'orthographe je crois pas (Blanche-Benveniste \& Willems 2007, p. 234). Cela ne fait que confirmer l'existence d'une dépendance macrosyntaxique par rapport à l'ancre.
} 
Les parenthétiques comme "Unités Illocutoires Associées 》

\subsection{Parenthétiques et ajouts}

Un autre indice de l'absence d'autonomie syntaxique des UIA concerne les contraintes qui s'imposent sur les ajouts dans ces unités. En effet, l'UIA refuse généralement l'ajout de circonstantiels qui en détaillent le sens. Une séquence comme celle en (16)c par exemple, est difficilement acceptable :

(16) a. Je pense depuis quelque temps qu'il va venir.

b. Il va venir, je pense.

c. *Il va venir, je pense depuis quelque temps.

Cette résistance aux ajouts s'explique sur des bases sémantiques: comme nous le verrons par la suite, les UIA ne servent pas à fournir des informations, mais plutôt à expliquer comment l'interlocuteur doit utiliser l'information qui est encodée par l'UI et elles ne permettent donc pas de circonstanciels spatio-temporels ${ }^{4}$.

De ce point de vue, les parenthétiques se distinguent assez clairement des parenthèses, comme (17)a, qui acceptent assez librement des changements de modalité (cf. (17)b) et des ajouts divers ( $c f .(17) c$.)

(17) a. vous voyez, euh alors ce que je regrette un petit peu < c'est que mes enfants (euh j'ai deux filles //) c'est que mes enfants eub ne soient pas dans le sport // (Gachet \& Avanzi 2008)

b. ce que je regrette un petit peu < c'est que mes enfants (vous saviez que j’ai deux filles? //) c'est que mes enfants euh ne soient pas dans le sport //

c. ce que je regrette un petit peu < c'est que mes enfants (j'ai deux filles qui viennent de terminer leurs études //) c'est que mes enfants ne soient pas dans le sport //

\subsection{Parenthétiques et incidence}

La parenthétique peut généralement être déplacée en position incidente de son ancre (cf. (18)). C'est d'ailleurs ce qui lui vaut l'appellation de parenthétique.

(18) a. Pierre est tombé, je crois.

b. Pierre, je crois, est tombé.

On peut penser que cette propriété est caractéristique des dites parenthétiques. Si l'on prend un enchaînement discursif tel que (19)a, on voit en (19)b qu'il n'est pas possible d'insérer la deuxième UI dans la première :

(19) a. Pierre est tombé. Marie est triste.

b. *Pierre, Marie est triste, est tombé.

4 L'exemple (2)c, qui contient l'UIA je me souviens maintenant, peut apparaitre comme un contrexemple à cette généralisation. Mais dans ce cas, maintenant ne remplit pas une fonction de modification de la description d'un état de choses, il fonctionne plutôt comme un marqueur de la mirativité ( $\approx$ mon souvenir est clair et cela me surprend). 
Mais si au lieu de prendre un enchaînement de cause à effet, nous prenons un enchaînement de type effet-cause, on voit qu'il est beaucoup plus naturel d'insérer une UI cause dans une UI effet :

(20) a. Pierre est tombé. Marie l'a poussé.

b. ?Pierre, Marie l'a poussé, est tombé.

Les UIA ne sont donc pas les seules UI à pouvoir venir s'insérer dans une autre UI.

A contrario, il existe un certain nombre de segments qui partagent la plupart des propriétés des parenthétiques (et seront donc couverts par notre définition des UIA), mais qui occupent obligatoirement une position initiale ou finale :

(21) a. tu prends tout droit là tu vois // (M006)

b. ?*tu prends, tu vois, tout droit là.

c. tu vois, tu prends tout droit là.

En conclusion, contrairement à l'usage qui prévaut pour les parenthétiques, nous ne retiendrons donc pas parmi les propriétés définitoires des UIA, le fait de pouvoir être en position incidente d'une autre UI.

\subsection{Parenthétiques et adjacence}

Remarquons que, à défaut d'être toujours insérable dans son ancre, l'UIA ne peut pas en être séparée. L'UIA doit toujours être adjacente à son ancre. Ainsi dans un enchaînement tel que (22)a, l'UIA je pense ne peut que porter sur la dernière UI, c'est-àdire qu'elle est paraphrasable par (22)b, mais pas par (22)c.

(22) a. eub dans la confusion $<+$ donc $<$ une eub une passante a dénoncé la jeune fille au livreur qui a couru après la jeune fille euh // les policiers sont arrivés en raison du du du vacarme je $\boldsymbol{p} \sim$ je pense // (M024)

b. Une passante a dénoncé la jeune fille au livreur qui a couru après la jeune fille. Je pense que les policiers sont arrivés en raison du vacarme.

c. Je pense qu'une passante a dénoncé la jeune fille au livreur qui a couru après la jeune fille et que les policiers sont arrivés en raison du vacarme.

C'est encore un indice d'une dépendance syntaxique entre l'UIA et son ancre.

Nous allons nous intéresser, dans les deux sections suivantes, à la relation sémantique que l'UIA entretient avec son ancre.

\section{Parenthétiques et dépendances sémantiques : la relation prédicative externe}

La paraphrase entre (7) et (8)a (j'ai deux itinéraires je crois je crois que j'ai deux itinéraires) permet de conclure que l'UR ancre j'ai deux itinéraires, à défaut d'être un actant syntaxique de la parenthétique je crois, en est quand même un argument sémantique. Il y a entre les deux UR de (7) une relation prédicative, le verbe principal de l'UR parenthétique prenant comme argument sémantique l'UR ancre ( $c$. aussi 
Iordanskaja \& Mel'čuk 2011). Le verbe CROIRE, même utilisé comme ici dans une parenthétique sans objet réalisé, reste intrinsèquement un prédicat binaire et son sens lexical ne peut être décrit sans faire explicitement référence à deux participants, celui qui croit quelque chose et ce qu'il croit. Il en va de même pour les autres verbes que l'on trouve dans des parenthétiques comme je pense, je trouve, on dirait, semble-t-il, tu vois, tu sais, etc.

Plus généralement, tout énoncé est constitué d'unités lexicales dont les sens fonctionnent comme des prédicats prenant comme argument les sens d'autres prédicats. Ceci fournit un graphe connexe de relations prédicat-argument qui constitue l'ossature de la représentation sémantique de l'énoncé (voir les représentations sémantiques de la Théorie Sens-Texte (Mel'čuk 1988, Kahane \& Mel'čuk 1999, Kahane 2003)), ainsi que des nombreux formalismes inspirés de la logique des prédicats de Frege, comme DRT (Kamp \& Reyle 1993) ou MRS (Copestake et al. 2005, Copestake 2009).

Les relations prédicatives sont usuellement réalisées par des relations microsyntaxiques, mais ce n'est pas toujours le cas. Il existe certaines unités lexicales, comme la locution CA ALLER, qui possèdent un argument sémantique qui est obligatoirement réalisé comme adnoyau, c'est-à-dire par une relation macrosyntaxique :

$$
\begin{aligned}
& \text { a. tes parents < ça va? } \\
& \text { b. *tes parents vont? }
\end{aligned}
$$

Cet argument ne peut être réalisé directement comme sujet, même s’il coréfère plus ou moins avec le sujet pronominal ça.

Il existe également des relations prédicatives qui vont au delà de la syntaxe et qui ont lieu entre deux UI indépendantes. Tel est le cas des connecteurs de discours, qui fonctionnent comme des prédicats binaires dont l'un des arguments est extérieur à l'UI où il se trouve :

a. Pierre est malade. Il ne pourra donc pas venir.

En (24)a, donc marque une relation de cause à effet entre être malade et ne pas powvoir venir, exactement comme le fait parce que en (24)b qui entretient clairement des relations de rection avec ses deux arguments (il est régi par pouvoir et régit être malade) :

b. Pierre ne pourra pas venir parce quil est malade.

Autrement dit, en (24)a, la prédication associée à donc reste insaturée à l'intérieur de l'UI à laquelle il appartient et trouve sa saturation dans une autre UI (Nølke 1990, Iordanskaja \& Mel'čuk 1995).

Les UIA, tout comme les connecteurs du discours, présentent une relation prédicative insaturée à l'intérieur de l'UI qu'elles forment et qui est saturée par l'UI ancre. Il est important de remarquer que cette propriété distingue les UIA d'autres parenthèses, telles que j'ai deux filles en (17)b. Effectivement, en (17)b, la parenthèse constitue une UI prédicativement saturée et la relation qu'elle établit avec l'UI dans laquelle elle s'insère n'est pas marquée par une relation prédicative contrôlée par une unité lexicale particulière, mais est une relation purement discursive entre deux UI. 


\section{Prédication du second ordre}

Une importante propriété sémantique des UIA semble découler du fait qu'elle constituent des relations prédicatives insaturées : les UIA constituent des prédications du second ordre. Nous allons voir dans le détail ce que cela signifie.

\subsection{Prédication du second ordre}

Les UIA ne contribuent pas de la même façon que les autres UI, et notamment que leur ancre, à la construction du savoir partagé des interlocuteurs. Alors que le contenu de l'ancre est un contenu susceptible d'être pris en charge, négocié entre les interlocuteurs, puis après vérification d'être inséré dans le savoir partagé, le contenu d'une UIA a une autre fonction : il sert à indiquer comment le contenu de l'ancre doit être interprété. Il est donc à la fois un contenu d'arrière plan, c'est-à-dire un contenu qui ne rentre pas dans le savoir partagé, et un contenu qui porte nécessairement sur d'autres contenus du discours, un contenu - dirait-on à l'instar de Mel'čuk (1993, p. 256) - du second ordre.

Cette distinction a été plusieurs fois proposée dans la littérature où l'on parle couramment d'une opposition entre contenu conceptuel ou vériconditionnel et contenu procédural (Ducrot 1986), entre niveau idéationnel et niveau textuel ou interpersonnel (Halliday 1976) ; ou encore entre prédication principale et prédication auxiliaire (Boye \& Harder 2007, 2009). Nous préférons formuler cette distinction comme une distinction entre un contenu informatif et un contenu de second ordre en ce qui regarde l'information. Ceci pour souligner que les UIA ne sont pas a priori privée d'un contenu vériconditionnel et conceptuel en tant que tel, même si ce contenu possède des propriétés différentes des contenus vériconditionnels ordinaires (cf. Ifantidou 1993).

Il existe une série de tests élaborés par différents auteurs qui permettent d'établir quand un signifié est un signifié informatif et quand il est un signifié de second ordre. Ces tests montrent d'une façon ou l'autre l'impossibilité pour l'interlocuteur de prendre en charge et de discuter le contenu d'un segment du second ordre.

Le premier de ces tests - repris de Bazzanella (1995) et Boye \& Harder (2009) consiste à vérifier la niabilité d'un segment, les segments informatifs étant niables, tandis que ceux du second ordre ne le sont pas :

(25) A: je l'ai toujours eu je crois (D201)

a. B: c'est pas vrai (que tu l'as toujours eu).

b. B: \#c'est pas vrai (que tu le crois).

Le second test est une déclinaison du premier et consiste à vérifier l'interrogeabilité d'un segment; les segments informatifs sont interrogeables, tandis que ceux du second ordre ne le sont pas ${ }^{5}$ :

\footnotetext{
${ }^{5} \mathrm{~B}$ peut produire une question comme Tu crois ? et donner l'impression d'interroger l'UIA de A. Il s'agit alors d'une UIA qui s'ancre sur l'UI produit par A et qui est possible, même si A ne produit pas l'UIA je crois. On remarque au passage qu'une UIA peut s'ancrer sur une UI énoncée par un autre locuteur.
} 
(26) A: je l'ai toujours eu je crois.

a. B: est-ce vrai (que tu l'as toujours eu)?

b. B: \# est-ce vrai (que tu le crois)?

Le troisième test - proposé par Ifantidou-Trouki (1993) - vérifie la possibilité de négocier le segment considéré. Une opération typique de la négociation entre locuteurs consiste pour le locuteur à prendre comme hypothèse ce qui a été asserté par son interlocuteur et d'en dériver une série de conséquences. Cette opération peut s'accomplir en insérant dans la protase d'une construction hypothétique, l'assertion de l'interlocuteur. Seuls les éléments informatifs peuvent être repris dans la protase, alors que ceux du second ordre en sont exclus :

(27) A: je l'ai toujours eu je crois.

a. B: si tu l'as toujours eu, tu l'as encore.

b. B: *si tu l'as toujours eu tu crois, tu l'as encore.

\subsection{Relation prédicative insaturée et prédication du second ordre}

Nous faisons l'hypothèse que la propriété sémantique des UIA de constituer des prédications du second ordre est liée au fait qu'elles constituent des relations prédicatives insaturées.

Dans un énoncé comme (7), que nous reprenons ici sous (28), l'UIA je crois prend comme argument sémantique $j$ 'ai deux itinéraires pas seulement en tant que syntagme, mais en tant qu'UI, c'est-à-dire en que syntagme porteur d'une force illocutoire :

\section{(28) j'ai deux itinéraires je crois //}

Il en résulte que la construction je crois est interprétée comme une prédication du second ordre portant sur l'énonciation et non pas comme un élément qui fournit des informations représentant directement la réalité métalinguistique.

Cette propriété rapproche les UIA des prédicats comme bon ou ben/bien. Ces éléments fournissent des informations qui viennent s'ajouter au savoir partagé lorsqu'ils fonctionnent comme modifieurs de leur argument sémantique (un bon repas, bien travailler), mais deviennent des prédicats du second ordre lorsqu'ils sont des marqueurs de discours dont l'argument sémantique n'est plus réalisé par une relation de rection :

(29) L1 : vous verrez euh tout est là // L2 : ok // L1 : voilà // L2 : ben je vous remercie beaucoup de votre attention (D008)

Nous allons préciser dans la section qui suit le rapprochement entre parenthétique et marqueur de discours que nous venons d'effectuer. 


\section{La classe des UIA}

\subsection{Définition des UIA}

Nous sommes maintenant en mesure de donner une définition des UIA.

Une UIA est un segment qui :

1) forme une UR, c'est-à-dire une unité rectionnelle maximale ;

2) possède une relation prédicative externe ${ }^{6}$ (cet argument externe est appelé l'ancre de l'UIA) ;

3) prend des modalités d'énonciation et possède une force illocutoire indépendante de celle de son ancre ; il forme donc une UI ;

4) entretient une dépendance macrosyntaxique avec son ancre et possède une syntaxe interne réduite, ne permettant pas par exemple n'importe quel ajout ou n'importe quelle modalité d'énonciation.

5) entretient une relation prédicative du second ordre avec son ancre.

Notre définition ne prévoit pas de critères concernant la forme interne des UIA ou leur position. De ce point de vue, elle se distingue de la définition des parenthétiques donnée par Schneider (2007, pp. 77-78). Schneider inclut parmi les parenthétiques toutes les clauses de son corpus de langues romanes qui :

1) sont basées sur un verbe fini ;

2) ne présentent pas un lien syntaxique marqué avec la clause ancre (Schneider parle en fait de clause hôte, mais nous avons expliqué pourquoi ce terme ne nous convenait pas);

\footnotetext{
${ }^{6}$ Nous avons trouvé dans le corpus de français parlé Rhapsodie constitué de 56 échantillons de 5 minutes en moyenne, trois exemples d'UR qui tout en étant saturées nous semblent être des UIA. Elles se trouvaient toutes dans le même échantillon, un extrait de flash d'information radiophonique.

a. un rendez-vous fixé vous l'avez dit après la forte mobilisation du 29 janvier dernier //

b. tout est bon vous le verrez avec Bertrand Vanier //

c. arrêtés hier matin vous le savez après trente six heures de cavale //
}

Nous pensons que ce sont quasiment des UIA, car le pronom peut être effacé sans modification essentielle du sens :

(ii) a. un rendez-vous fixé vous avez dit après la forte mobilisation du 29 janvier dernier //

b. tout est bon vous verrez avec Bertrand Vanier //

c. arrêtés hier matin vous savez après trente six heures de cavale //

Nous attribuons la présence du pronom à un phénomène de surcorrection dû au registre particulier de cet échantillon. Il nous semble aussi que, dès que le pronom est présent et que l'UR est saturée, elle devient plus facilement négociable (ce qui n'est pas vraiment un problème pour le locuteur dans ce contexte particulier d'énonciation, où il ne peut être interrompu). 
3) occurrent au moins une fois en position médiane et une fois en position finale ;

4) interrompent au moins un fois en position médiane une relation syntaxique étroite (c'est-à-dire une relation de rection) ;

5) sont ancrées sur une clause structurellement autosuffisante (c'est-à-dire syntaxiquement autonome);

6) ont une valence insaturée ;

7) sont ancrées sur une clause dont on peut inférer l'argument manquant.

Nous allons comparer nos deux définitions. Nous montrerons d'abord que elles définissent des classes de syntagmes assez différents, puis nous discuterons de comment évaluer la pertinence respective de deux définitions qui ne définissent pas la même chose.

\subsection{UIA et incises de citation}

Considérons une incise de citation telle que :

(30) a. «Casse-toi, pauv’ con! ! dit-il.

Schneider inclut les incises de citation dans sa définition. Nous ne les incluons pas parmi les UIA pour plusieurs raisons. Premièrement, bien que la relation syntaxique ne soit pas marquée, nous considérons qu'il s'agit d'une relation de rection (ce qui fait qu'elle vérifie la propriété (1) de Schneider mais pas notre propriété (1)). Le clivage n'est pas directement possible à partir de la forme (30)a, mais elle l'est à partir de la forme (30)b, où la rection est indéniable, comme le montre (30)c.

b. Il a dit : «Casse-toi, pauv' con!».

c. C’est bien « Casse-toi, pauv' con!» qu'il a dit.

Nous pensons que (30)a est essentiellement une forme inversée de (30)b appartenant à un registre littéraire (voir Danlos et al. (2010) pour une thèse opposée). Le fait d'ailleurs que les incises de citation ne figurent pas à l'oral spontané ${ }^{7}$ est une deuxième propriété qui les distingue nettement des UIA, toutes très fréquentes à l'oral.

La troisième propriété pour laquelle nous considérons que les incises de citations ne sont pas des UIA est que le verbe de citation peut se combiner librement avec divers ajouts :

d. «Casse-toi, pauv’ con!» décida-t-il de dire à la personne qui lui faisait face.

Enfin, le verbe de citation peut recevoir n'importe quelle modalité d'énonciation et n'importe quel ajout :

d. Ne dis plus jamais : «Casse-toi, pauv’ con!»!

e. A-t-il vraiment dit l'autre jour : «Casse-toi, pauv’ con!»?

\footnotetext{
7 On trouve néanmoins un grand nombre d'incises de discours dans un registre oral très particulier (et non spontané) qui est celui des flashs info. Voir la note précédente à propos de ce même registre de discours.
} 


\subsection{UIA non incidentes}

La décision de ne pas inclure a priori de critères positionnels dans notre classification permet de retenir parmi les UIA une série de syntagmes typiquement antéposés à leur ancre comme tu verras, je veux dire, comment dire ou écoutez et qui sont exclus de la classe des parenthétiques de Schneider.

(31) a. et tu verras c'est un arrêt de tram aussi // (M006)

b. je veux dire là ça me laisse rêveur Paul Valéry en zep // (D001)

c. c'est comme \& // comment dire il il y a certains métiers où il $\mathrm{f} \sim$ il faut être né eub [vous voyez ce que je veux dire //] // il faut être né psychiatre pour être psychiatre // (D006)

d. A : je voudrais reprendre euh des phrases de Marcel Achard // et vous me direz si vous êtes d'accord // B : oui // A: Marcel Achard écrivait [...] //

B: écoutez vous m'avez demandé si je suis d'accord avec Marcel Achard // je suppose que vous connaissez Marcel Achard // c'est un homme incapable de dire quoi que ce soit de désagréable sur qui que ce soit // je ne vois pas pourquoi j'aurais fait exception à cette règle // (D201)

\subsection{UIA et marqueurs du discours}

Notre décision de ne pas utiliser de critères formels internes (et en particulier de ne pas imposer la présence d'un verbe) nous amène à inclure dans les UIA non seulement des UR verbales, mais aussi des UR étymologiquement verbales (comme voilà ou tiens) utilisées comme marqueurs de discours :

a. là < sur la place de Verdun < il y a mb la Préfecture voilà // (M003)

b. Tiens, c'est rigolo, un coup j'ai 10 réponses visibles, un coup j'en ai que 5 ! Pourquoi ? Bug ou Censure? (site d'annonces automobiles)

ainsi que les marqueurs de discours issus d'autres parties du discours qui sont susceptibles de recevoir une modalité d'énonciation (adjectifs comme bon, adverbes comme ben (issu de bien), pronoms comme quoi, interjections pures comme hein ou ob) :

(33) a. et c'est vrai que bon habitant dans le centre de Paris eub $<$ les écoles sont de très bon niveau hein je veux dire // (D002)

b. et euh et ces rails du tram < eh ben je vais je vais les longer // (M016)

c. tu arrives place aux Herbes avec une une sorte $\{$ de halle $\mid$ quoi de de de structure métallique \} // (M001)

d. ça doit être \{cinq cent | peut-être $\boldsymbol{o h}$ un petit kilomètre\} plus loin // (M013)

Certains arguments nous amènent à faire l'hypothèse que ces marqueurs de discours sont bien des UIA.

1) Ils ne sont pas régis et forment donc des UR.

2) Ce sont des prédicats ayant comme argument sémantique l'UI ancre. Certains sont issus de formes verbales insaturées (voilà, tiens), tandis que d'autres sont issus de 
lexèmes adjectivaux ou adverbaux (bon, ben) qui dans leur emploi canonique fonctionnent comme des modifieurs d'un élément qui est leur argument sémantique (dans un bon fautenil, bon dénote une propriété qui s'applique au fauteuil, de même que dans bien manger, bien est une propriété qui s'applique au procès manger).

3) Ils possèdent leur propre force illocutoire. Par exemple, hein peut être interrogatif, tiens exclamatif et bon est assertif et ceci indépendamment de l'UI ancre, qui peut recevoir une modalité d'énonciation différente.

4) Ils n'acceptent aucune modification, ni ajout, ni changement de force illocutoire.

5) Ce sont intrinsèquement des prédicats du second ordre. Ils s'interprètent seulement par rapport à l'UI sur laquelle ils s'ancrent. Il est absolument impossible à l'interlocuteur de considérer qu'ils lui sont adressés en tant que tel et d'appuyer sa réponse sur leur contenu.

Notre classification permet ainsi de reconnaitre et d'interpréter formellement la parenté, plusieurs fois soulignée dans la littérature sur les marqueurs de discours, entre ceux-ci et les parenthétiques (Brown \& Levinson 1978, Ostman 1981, Holmes 1986, Schiffrin 1987, Bazzanella 1995, Aijmer 2002, Karkkainen 2003). Nous considérons non seulement que ces marqueurs de discours ont la même fonction que les parenthétiques, comme il a été plusieurs fois remarqué et comme nous allons continuer à le montrer, mais aussi qu'ils possèdent avec l'UI qui leur sert d'ancre la même relation macrosyntaxique.

\section{Fonctions des UIA}

Nous allons apporter une nouvelle justification au fait de considérer la classe des UIA en précisant le rôle que jouent les UIA dans la langue.

Rappelons que les UIA sont des éléments qui : (i) établissent une relation prédicative du second ordre avec leur ancre, (ii) sont intégrés au niveau macrosyntaxique et (iii) constituent des UI en soi, susceptibles d'accepter une modalité d'énonciation. Le français dispose d'autres instruments qui permettent de fournir des prédications du second ordre : les recteurs faibles (Blanche-Benveniste (1989), Blanche-Benveniste \& Willems (2007)) ( $f$. (34)a) ou les adverbes d'énonciation qui se trouvent en adnoyau (cf. (34)b).

(34) a. je pense que [à l'intérieur de Paris $<+e u b$ globalement $<e u b$ les les écoles sont sont d'un bon niveau ] voilà // (D002)

b. en tout cas $<$ la recherche fondamentale $<$ elle elle reste libre $>$ heureusement $/ /$ (D005)

Il a été souvent reconnu que ces éléments, comme les UIA, servent à donner des instructions pour l'interprétation de l'information fournie, plutôt qu'à fournir des informations qui s'ajoutent au savoir partagé. Il faut donc éclaircir en quoi les UIA se distinguent de ces éléments et quelle fonction particulière elles accomplissent.

La différence entre les prédicats du second ordre intégrés et les UIA réside dans le fait que les UIA constituent des UI séparées de leur ancre. Cette propriété permet aux UIA d'être interprétées comme des actes linguistiques à part entière qui peuvent 
reprendre ou anticiper une portion du discours en cours pour en préciser certains aspects. Certains auteurs ont défini cette fonction comme une fonction de commentaire métalinguistique sur le discours en cours (Espinal 1991, p. 760, Brinton 2007).

Cette fonction qui a été reconnue également pour les marqueurs discursifs (Schiffrin 1987) peut se préciser, dans le cas des UIA verbales, dans un nombre de fonctions plus particulières, souvent reconnues dans la littérature (Blanche-Benveniste \& Willems 2007, Ifantidou 1993, Schneider 2007, Beyssade \& Marandin 2006, 2009).

Il nous intéresse de souligner que, à côté des fonctions de modalisation épistémique (35)a ou évidentielle (35)b :
a. bon je l'ai toujours eu je crois // (D201)
b. $\boldsymbol{t u}$ as $\boldsymbol{v u}$ il répond d'abord // et il comprend après // (D207)

et de précision (35)c ou de reformulation (35)d de la force illocutoire (Urmson 1952, Venier 1991, Ifantidou 1993, Schneider 2007, Beyssade \& Marandin 2006, 2009) :

c. en ce sens < c'est j'insiste une grande loi de régulation // (allocution de Frédéric Mitterand au Centre National du Livre)

d. $+30 \%$ de dégat crit avant d'avoir la lame d'inf, ca fait toujours du bien tu crois pas? (forum, League of Legends Community)

les UIA accomplissent une fonction intrinsèquement métalinguistique, qui n'est pas partagée par les recteurs faibles ou les modaux en adnoyau, qui est celle de commentaires sur un choix lexical ou un choix de formulation pour un syntagme. Par exemple, si nous reprenons l'exemple (1)a reproduit en (36), nous voyons que l'UIA je crois a pour rôle d'indiquer que le locuteur hésite dans la formulation du syntagme qui suit $^{8}$ :

(36) nous avons attendu je crois sept ou huit heures dans la gare parce que tous les officiers allemands tout l'état major allemand rentraient à Paris // (D003)

Cette fonction de commentaire sur la formulation se produit quand l'UIA porte sur un syntagme ou sur un lexème et souvent l'élément dans la portée de l'UIA se trouve dans un entassement ${ }^{9}$. Dans ce cas, la position de l'UIA dans l'entassement semble saillante.

\footnotetext{
${ }^{8}$ On notera au passage que l'UIA en question se trouve alors en position incidente de l'UI complète, mais que vis-à-vis de l'élément qu'elle commente, dont on peut considérer qu'il s'agit de son ancre véritable, elle occupe une position adjacente. La position incidente n'est donc qu'une conséquence de la possibilité pour certaines UIA de commenter les choix lexicaux.

${ }^{9}$ Les entassements reformulatifs sont un autre indice de la difficulté à trouver la bonne formulation et il n'est pas surprenant qu'ils se combinent avec les éléments qui commentent les formulations. Il existe encore une autre construction remarquable, la greffe (Deulofeu 1999), qui se produit en cas de difficulté de formulation. On dit qu'il y a greffe lorsqu'une UI est produite en lieu et place d'un syntagme régi (voir aussi l'exemple (31)c) :

(i) vous suivez la ligne du tram qui passe vers la [je crois que c'est une ancienne caserne je crois //] voilà // (M003)
}

Il existe à notre avis un lien assez étroit entre greffe et UIA. Non seulement, comme dans l'exemple précédent, la greffe peut se combiner avec une UIA, mais les deux sont plus ou moins 
Une même UIA, comme on va dire, peut avoir une fonction de marqueur de l'approximation si elle modifie la première couche d'un entassement (cf. (37)a), et une fonction de marqueur de la reformulation si elle modifie la dernière couche d'un entassement (cf. (37)b) :

(37) a. je tombe \{ sur la place Sainte-Claire on va dire | là où il y a la halle \}// (M014)

b. oui bon alors euh \{pour ce qui est de \& | pour ce qui est \{du livre jeunesse $\mid$ on va dire du livre de détente $\}$ (D004)

Et effectivement, la fonction d'approximation lexicale semble régulièrement associée aux UIA qui modifient la première couche d'un entassement et celle de reformulation aux UIA qui modifient la dernière couche :

a. il y a maintenant $p f f$ peut-être au moins \{ douze ans je pense hein $\mid$ douze treize ans (D004)

b. c'est la \{ première| ou enfin non euh \{deuxième | ou troisième $\}$ troisième il me semble $\}$ à droite (M009)

En résumé, nous pensons que la fonction générale des UIA est une fonction de commentaire métalinguistique, qui peut se spécifier dans un certain nombre de fonctions particulières, parmi lesquelles on remarquera une fonction de marquage de la formulation. Notre approche se distingue, de ce point de vue, des approches qui attribuent aux parenthétiques la fonction générale d' "apporter un type de précaution », d'atténuer pour des raisons diverses une assertion ou une partie d'une assertion (Willems \& Blanche-Benveniste 2010, Schneider 2007).

\section{Conclusion}

Dans cet article, nous avons avancé une réponse à la question du niveau d'intégration des parenthétiques en les intégrant au niveau de la structure macrosyntaxique de l'énoncé. L'approche macrosyntaxique, comme on l'a vu, permet de prendre en compte des contraintes distributionnelles de nature syntaxique des UIA verbales sans qu'on soit pour autant obligé de parler de rection. Ainsi la relation sémantique entre les parenthétiques et l'ancre n'est-elle pas simplement inférée à un niveau pragmatique, comme le serait celle entre deux phrases indépendantes, mais elle est marquée par une relation prédicative qui possède une réalisation syntaxique particulière (même si cette relation syntaxique se situe au delà de la rection) et qui a une fonction sémantique spécifique, celle de commentaire métalinguistique.

Cette approche nous a amenés à introduire une nouvelle classe d'unités (macro)syntaxiques: les unités illocutoires associées. Comme on l'a vu cette classe englobe les parenthétiques, à l'exception des incises de discours. Il est alors légitime de ce demander en quoi les UIA forment, du point de vue théorique, une classe plus légitime ou non que la classe plus traditionnelle des parenthétiques.

interchangeables et on pourrait aller jusqu’à considérer que les UIA qui s'ancrent sur des syntagmes fonctionnent comme des sortes de greffes dont le noyau serait le syntagme ancre (ii) :

(ii) vous suivez la ligne du tram qui passe vers [une ancienne caserne $>$ je crois //] // 
L'objet d'une classification est d'obtenir un classement raisonné des différentes espèces d'un ensemble. La caractérisation d'une classe ne peut donc s'apprécier qu'au sein d'une classification plus générale d'un certain type d'objets. Prenons l'exemple des parties du discours: il s'agit d'une classification des lexèmes et chaque partie du discours regroupe un grand nombre de lexèmes qui possèdent un certain nombre de propriétés communes que ne possèdent pas les lexèmes en dehors de cette classe. Cette classe apparaît ainsi dans un grand nombre de règles de la grammaire et c'est ce qui fait sa légitimité.

Notre définition des UIA entre dans une tentative de classification générale des unités syntaxiques et plus précisément des unités de rection (UR). Rappelons que les UR sont les unités maximales de la microsyntaxe, c'est-à-dire des segments dont les éléments sont liés par des relations de rection, mais n'entretiennent pas de relation de rection au delà. Si la classification des unités syntaxiques de la microsyntaxe, c'est-àdire les syntagmes au sein de l'UR, est une question qui a fait l'objet de très nombreuses études depuis les premiers travaux des distributionnalistes, la classification des unités syntaxiques de la macrosyntaxe, c'est-à-dire des regroupements d'UR, est un sujet encore jeune. Parmi les UR, certaines forment des noyaux, c'est-à-dire possèdent une force illocutoire et peuvent recevoir librement des modalités d'énonciation. D'autres UR, comme les adnoyaux, ne portent pas de force illocutoire et se raccrochent à un noyau par le fait d'être dans la portée de la force illocutoire de ce dernier. En définissant les UIA, nous regroupons une classe d'UR qui possèdent des propriétés à la fois différentes des noyaux et des adnoyaux, puisqu'elles possèdent à la différence des adnoyaux une certaine force illocutoire, mais n'ont pas l'indépendance des noyaux (puisqu'elles ne peuvent pas recevoir n'importe quelle modalité d'énonciation).

De ce point de vue, notre démarche diffère de l'approche traditionnelle sur les parenthétiques qui cherche avant tout à classer les constructions verbales qui se trouvent en incidence d'une autre construction verbale. De telles études se situent généralement dans une approche du texte vu comme une succession de constructions verbales qui forment autant de phrases ${ }^{10}$ et la position incidente de certaines constructions verbales est de ce point de vue problématique.

En limitant l'étude aux constructions verbales en position incidente, on ne peut pleinement saisir la nature de la relation qui unit une parenthétique à son ancre. Nous pensons qu'il faut partir plutôt d'une classification des relations entre unités syntaxiques, qui nous amène à la définition des UR, puis des relations macrosyntaxiques entre UR. Et c'est ensuite que l'on peut éventuellement se poser la question de savoir pourquoi certaines relations macrosyntaxiques permettent à une UR d'avoir une position incidente, question que nous n'avons fait qu'effleurer ici.

\footnotetext{
${ }^{10}$ De nombreux travaux ont bien sûr montré en quoi cette conception du texte est problématique, en particulier lorsqu'on considère la langue parlée. On se rapportera notamment aux travaux précurseurs de l'école d'Aix, dont il a été déjà question dans notre introduction. La notion de phrase présuppose en général une certaine coïncidence des notions d'UR et d'UI ou au moins une relation d'inclusion entre les deux notions. Or comme nous l'avons montré, il existe des UI composées de plusieurs UR, mais aussi des UR qui vont au delà de l'UI (cf. Benzitoun et al. 2010 pour un développement).
} 
Une des conséquences de notre approche est d'avoir proposé qu'on puisse regrouper au sein d'une même classe les parenthétiques, qui forment une partie des UIA verbales (l'autre partie étant les UIA verbales qui occupent nécessairement une position initiale ou finale d'une UI), et certains marqueurs de discours qui constituent des UIA non verbales. Les UIA et ce type de marqueurs du discours constituent effectivement des unités illocutoires qui entretiennent des relations de prédication externe du second ordre avec leurs ancres. Cette caractérisation formelle justifie la parenté plusieurs fois remarquée dans la littérature fonctionnelle entre ces deux classes d'objets linguistiques.

\section{Remerciements}

Nous remercions Lida Iordanskaja et Igor Mel'čuk d'avoir relu et commenté notre article. Ils ont eux-mêmes écrit un article à la suite de ces échanges sur la sémantique des UIA. Nous remercions également l'ensemble des participants à l'annotation syntaxique du corpus Rhapsodie et particulièrement José Deulofeu qui a également relu l'article. C'est au cours des séances de discussion de ce groupe qu'est apparu progressivement l'idée que certains segments n'étaient ni réellement des noyaux, ni des adnoyaux typiques. Nous remercions également les relecteurs de la revue, ainsi que Julie Glikman et Mathieu Avanzi de nous avoir proposé d'écrire cet article. 


\section{RÉFÉRENCES BIBLIOGRAPHIQUES}

Aijmer, K. (2002), English Discourse Particles, Evidence from a Corpus, Amsterdam/Philadelphia, Benjamins.

BAzzANElla, C. (1995), «I segnali discorsiv i », in L. Renzi, G. Salvi, A. Cardinaletti (éds), Grande grammatica italiana di consultazione, vol. III, Bologna, Il Mulino, pp. 225-257.

Benzitoun, C., Dister, A., Gerdes, K., Kahane, S., Pietrandrea, P. \& Sabio, F. (2010), «Tu veux couper là faut dire pourquoi. Propositions pour une segmentation syntaxique du français parlé », Actes du Congrès Mondial de Linguistique Française, La Nouvelle Orléans.

Beyssade, C., \& Marandin, J.-M. (2006), «The Speech Act Assignment Problem Revisited: Disentangling Speaker's Commitment from Speaker's Call on Addressee », in Bonami O. \& Cabredo-Hoffher P. (éds), Empirical Studies in Syntax and Semantics, nº 6 , pp. 37-68.

Beyssade, C., \& Marandin, J.-M. (2009), «Commitment : une attitude dialogique », Langue Française, n¹62, pp. 89-107.

Blakemore, D. (2006), «Divisions of labour: The analysis of parentheticals », Lingua, n¹66, pp. $1670-1687$.

BlanCHE-BenVEniste, C. (1989), «Constructions verbales en incise et rection faible des verbes », Recherches sur le français parlé, $\mathrm{n}^{\circ} 9$, pp. 53-73.

Blanche-Benveniste, C., Bilger, M., Rouget, C. \& VAn Den Eynde, K., (1990), Le français parlé. Etudes grammaticales, Paris, Editions CNRS.

Blanche-Benveniste, Cl.., \& Willems, D. (2007), «Un nouveau regard sur les verbes faibles », Bulletin de la société linguistique de Paris, n¹02 (1), pp. 217-254.

BOYE, K., \& HARDER P. (2007), « Complement-taking predicates. Usage and linguistic structure », Studies in Language, $\left.\mathrm{n}^{\circ} 31 / 3\right)$, pp. 569-606.

BOYE, K., \& HARDER P. (2009), «Evidentiality Linguistic categories and grammaticalization Functions of Language », Language, n¹6/1), pp. 9-43.

BRINTON, L .J. (2007), The Comment Clause in English, Cambridge, Cambridge University Press.

Brown, P., \& Levinson, S. (1978), «Universals in Language Use: Politeness Phenomena. », in Goody E. (éd.) Questions and Politeness: Strategies In Social Interaction, Cambridge, Cambridge University Press, pp. 56-310.

Cresti, E. (2000), Corpus di italiano parlato, Florence, Accademia della Crusca.

Copestake, A., Flinckinger, D., Sag I. \& Pollard C. (2005), « Minimal Recursion Semantics: An introduction ", Journal of Research on Language and Computation, $\left.\mathrm{n}^{\circ} 3 / 2-3\right)$, pp. 281-332.

Copestake, A. (2009), «Slacker Semantics: Why Superficiality, Dependency and Avoidance of Commitment can be the Right Way to Go », Proceedings of the 12th EACL, Athènes, pp. 1-9.

Danlos, L., SAgot, B. \& Stern, R. (2010), « Analyse discursive des incises de citation », Actes du Congrès Mondial de Linguistique Française, La Nouvelle Orléans.

Dehé, N. \& Kavalova, Y. (2006), «The syntax, pragmatics, and prosody of parenthetical what ", English Language and Linguistics, n¹0, pp. 289-320. 
ESPINAL, T. (1991), «The representation of disjunct constituents », Language, n67/4, pp. 726-762.

DEulofeu, J. (1999), Recherches sur les formes de la prédication dans les énoncés assertifs en français contemporain (le cas des énoncés introduits par le morphème que), Thèse d'état, Université Paris 3.

Ducrot, O. (1984), Le dire et le dit, Paris, Minuit.

GACHET, F. \& AvANZI, M (2008). «La prosodie des parenthèses en français spontané »Verbum, $\mathrm{n}^{\circ} 30 / 1$, pp. $53-84$.

Gerdes, K. \& Kahane, S. (2009), «Speaking In Piles: Paradigmatic Annotation Of French Spoken Corpus », Proceedings of the Fifth Corpus Linguistics Conference, Liverpool.

Haegeman, L. (1988), «Parenthetical adverbials: the radical orphanage approach », Chiba, S. (éd.) Aspects of Modern English Linguistics, Tokyo, Kaitakushi, pp. 232-254.

Haldiday, M.A.K. (1976), System and Function in Language. Selected Papers edited by G.R. Kress, Oxford, Oxford University Press.

Holmes, J. (1986), «Functions of you know in women's and men's speech », Language in Society, $\mathrm{n}^{\circ} 15$, pp. 1-21.

Ifantidou, E. (1993), «Parentheticals and relevance », UCL Working Papers in Linguistics, 5, pp. 193-210.

IfANTIDOU-Trouki E. (1993), «Sentential adverbs and relevance », Lingua, n 90, pp. 69-90.

IORDANSKAJA, L. \& MEL'CUK, I (1995), «Traitement lexicographique de deux connecteurs textuels du français contemporain : EN FAIT vs EN RÉALITÉ » in Bat-Zeev Shyldkrot, H. \& Kupferman, L. (réd.), Tendances récentes en linguistique française et générale (volume dédié à David Gaatone), Amsterdam/Philadelphia: Benjamins, pp. 211-236.

Iordanskaja, L. \& MeL’CuK, I (2011), « Illocutive Parenthetical Verbs in Russian », Proceeding of the Fifth Conference on Meaning-Text Theory, Barcelone, pp. 120-133.

Kahane, S. (2003), «Meaning-Text Theory ». in Ágel, V., Eichinger, L. M., Eroms, H. W., Hellwig, P., Heringer, H. J., Lobin, H. (éds), Dependency and Valency, Handbooks of Linguistics and Communication Sciences, n²5 (1-2), Berlin/NY, De Gruyter.

KAHANE, S. \& MEL'CUK, I. (1999), «La synthèse sémantique ou la correspondance entre graphes sémantiques et arbres syntaxiques - Le cas des phrases à extraction en français contemporain » T.A.L., $\mathrm{n}^{\circ} 40 / 2$, pp. 25-85.

KAHANE, S. (à par.), «De l'analyse en grille à la modélisation des entassements », in Caddeo, S., Roubaud, M.-N., Rouquier, M., Sabio, F., Hommage à Claire Blanche-Benveniste, Presses de l'université de Provence.

KAHANe, S. \& Pietrandrea, P. (à par.), «Typologie des entassements en français ».

Kamp, H. \& ReYle, U. (1993), From Discourse to Logic. Introduction to Model-theoretic Semantics of Natural Language, Formal Logic and Discourse Representation Theory, Dordrecht Kluwer Academic Publishers.

KÄRKKÄINEN, E. (2003), Epistemic stance in English conversation. A description of its interactional functions, with a focus on I think, Amsterdam, Benjamins.

MeL'CuK, I. (1988), Dependency Syntax: Theory and Practice, Albany, SUNY.

MEL’CUK, I. (1993), Cours de morphologie générale, Vol. 1, Montréal, PUM / Paris, CNRS. 
Sylvain Kabane et Paola Pietrandrea

NøLKE, H. (1990), «Il y a connecteurs et connecteurs : la connexion analytique et synthétique en français moderne », in Stammerjohan H. (éd.), Analyse et synthèse dans les langues slaves et romanes, Tübingen, Gunther Narr, pp. 41-48.

Ostman, J.-O. (1981), "You know». A Discourse-functional Approach. Amsterdam/Philadelphia, Benjamins.

Ross, J. R. (1973), «Slifting », in M. Gross, M. Halle, M. P Schützenberger (éds), The Formal Analysis of Natural Language, Berlin, Mouton, pp. 133-169.

SABIO, F. (2006), « L'antéposition des compléments dans le français contemporain : l'exemple des objets directs », in K. Gerdes \& C. Muller (éds), Ordre des mots et topologie de la phrase française, Lingvisticae Investigationes, $\mathrm{n}^{\circ} 29 / 1$, pp. 173-182.

Schelfhout, C., Arno Coppen, P. \& Oostdijk, N. (2004), «Finite Comment Clauses in Dutch: A Corpus-based Approach », Journal of Germanic Linguistics, n¹6, pp. 331-349

SCHIFFrin, D. (1987), Discourse Markers. Cambridge, Cambridge University Press.

SCHNEIDER, S. (2007), Reduced parenthetical clauses. A corpus Study of Spoken French, Italian and Spanish, Amsterdam/Philadelphia, Benjamins.

Urmson, J. (1952), « Parenthetical verbs », Mind, n61, pp. 480-496.

Venier, F. (1991), La modalizzazione assertiva, avverbi modali e verbi parentetici. Milan, Franco Angeli.

Willems, D. \& BLANCHE-BENVENiste, C. (2010), « Verbes ‘faibles’ et verbes à valeur épistémique en français parlé : il me semble, il parait, j’ai l'impression, on dirait, je dirais », in Iliescu, M. et al. (éds), Actes du XXV Congrès International de Linguistique et de Philologie Romanes, Innsbruck, septembre 2007, Tome IV, De Gruyter, pp. 565-579. 